\title{
Mitochondrial markers predict recurrence, metastasis and tamoxifen-resistance in breast cancer patients: Early detection of treatment failure with companion diagnostics
}

\author{
Federica Sotgia ${ }^{1}$, Marco Fiorillo ${ }^{1,2}$ and Michael P. Lisanti ${ }^{1}$ \\ ${ }^{1}$ Translational Medicine, School of Environment \& Life Sciences, University of Salford, Greater Manchester, United Kingdom \\ 2 The Department of Pharmacy, Health and Nutritional Sciences, The University of Calabria, Cosenza, Italy \\ Correspondence to: Michael P. Lisanti, email: michaelp.lisanti@gmail.com \\ Federica Sotgia, email: fsotgia@gmail.com \\ Keywords: mitochondria, mitochondrial biogenesis, biomarkers, treatment failure, relapse \\ Received: May 23, $2017 \quad$ Accepted: June 16, $2017 \quad$ Published: July 27, 2017 \\ Copyright: Sotgia et al. This is an open-access article distributed under the terms of the Creative Commons Attribution License 3.0 (CC BY \\ 3.0), which permits unrestricted use, distribution, and reproduction in any medium, provided the original author and source are credited.
}

ABSTRACT

Here, we used a data-mining and informatics approach to discover new biomarkers of resistance to hormonal therapy in breast cancer. More specifically, we investigated whether nuclear-encoded genes associated with mitochondrial biogenesis can be used to predict tumor recurrence, distant metastasis and treatment failure in high-risk breast cancer patients. Overall, this strategy allowed us to directly provide in silico validation of the prognostic value of these mitochondrial components in large and clinically relevant patient populations, with $>15$ years of follow-up data. For this purpose, we employed a group of 145 ER(+) luminal A breast cancer patients, with lymph-node (LN) metastasis at diagnosis, that were treated with tamoxifen, but not any chemotherapy agents. Using this approach, we identified $>60$ new individual mitochondrial biomarkers that predicted treatment failure and tumor recurrence, with hazard-ratios (HR) of up to $4.17(p=2.2 \mathrm{e}-07)$. These include mitochondrial chaperones (HSPD1, HSPA9), membrane proteins (VDAC2, TOMM70A) and anti-oxidants (SOD2), as well as 18 different mitochondrial ribosomal proteins (MRPs) and >20 distinct components of the OXPHOS complexes. In addition, we combined 4 mitochondrial proteins (HSPD1, UQCRB, MRPL15, COX17), to generate a compact mitochondrial gene signature, associated with a HR of $5.34(p=1 \mathrm{e}-09)$. This signature also successfully predicted distant metastasis and was effective in larger groups of $\operatorname{ER}(+)(N=2,447)$, basal $(N=540)$ and HER2 $(+)(N=193)$ breast cancers. It was also effective in all breast cancers $(N=3,180)$, if considered together as a single group. Based on this analysis, we conclude that mitochondrial biogenesis should be considered as a new therapeutic target for overcoming tumor recurrence, distant metastasis and treatment failure in patients with breast cancer. In summary, we identified individual mitochondrial biomarkers and 2 compact mitochondrial gene signatures that can be used to predict tamoxifen-resistance and tumor recurrence, at their initial diagnosis, in patients with advanced breast cancer. In the long-term, these mitochondrial biomarkers could provide a new companion diagnostics platform to help clinicians to accurately predict the response to hormonal therapy in $\mathrm{ER}(+)$ breast cancer patients, facilitating more personalized and effective treatment. Similarly, these mitochondrial markers could be used as companion diagnostics, to determine which breast cancer patients would benefit most from clinical treatments with mitochondrially-targeted anti-cancer therapeutics. Finally, we also showed that these mitochondrial markers are superior when directly compared with conventional biomarkers, such as Ki67 and PCNA. 


\section{INTRODUCTION}

Treatment failure, due to drug resistance, still remains a major obstacle for more effective anti-cancer therapy and personalized medicine [1-9]. In estrogenreceptor-positive $(\operatorname{ER}(+))$ breast cancer, approximately 40 -to- $50 \%$ of patients eventually develop tamoxifenresistance [5-9]. Importantly, the five-year survival rate following tamoxifen-resistance is less than $20 \%$ [1-5]. Unfortunately, tamoxifen-resistance often manifests itself as tumor recurrence and/or distant metastasis. As such, resistance to endocrine therapy is a critical factor that still limits the efficacy of breast cancer treatment. Thus, better biomarkers and companion diagnostics are needed for the early detection of patients that will likely fail hormonal therapy [5-9].

Here, we set out to test the hypothesis that individual markers of mitochondrial biogenesis and OXPHOS may have prognostic value in the early identification of tamoxifen-resistant patients at diagnosis, up to 15 years before the onset of tumor recurrence and distant metastasis. For this purpose, we performed outcome analysis on $>400$ nuclear mitochondrial gene transcripts.

Our results indicate that $>60$ different mitochondrial markers can be used individually or in combination, as short signatures, to predict tumor recurrence in tamoxifentreated breast cancer patients. As a consequence, we discuss the possibility that mitochondria should be therapeutically targeted, to overcome resistance to hormonal therapy, and to prevent tumor recurrence and distant metastasis. In accordance with this idea, metformin (a mitochondrial complex I inhibitor) has been previously shown to overcome tamoxifen-resistance in $\mathrm{ER}(+)$ cell culture models, which mimic the tumor microenvironment by the addition of stromal fibroblasts [9-11].

Interestingly, mitochondrial markers also showed prognostic value in different sub-groups of ER(-) breast cancer patients [12].

\section{RESULTS}

\section{Establishing the prognostic value of conventional markers in the patient population}

To identify new potential biomarkers of tamoxifen-resistance, here we used publically available transcriptional profiling data from the tumors of breast cancer patients that were treated with tamoxifen, but did not receive any chemotherapy. For this purpose, we selected high-risk patients that were lymph-node positive at diagnosis, and we focused on the luminal A subtype, which represents the most common form of $\operatorname{ER}(+)$ breast cancers $(N=145$ patients) (Figure 1$)$.

As proliferative markers are often used as the primary endpoint in clinical trials, we first assessed the prognostic value of Ki67 and PCNA, in this patient population. Table 1 and Figure $2 \mathrm{~A}$ both show the

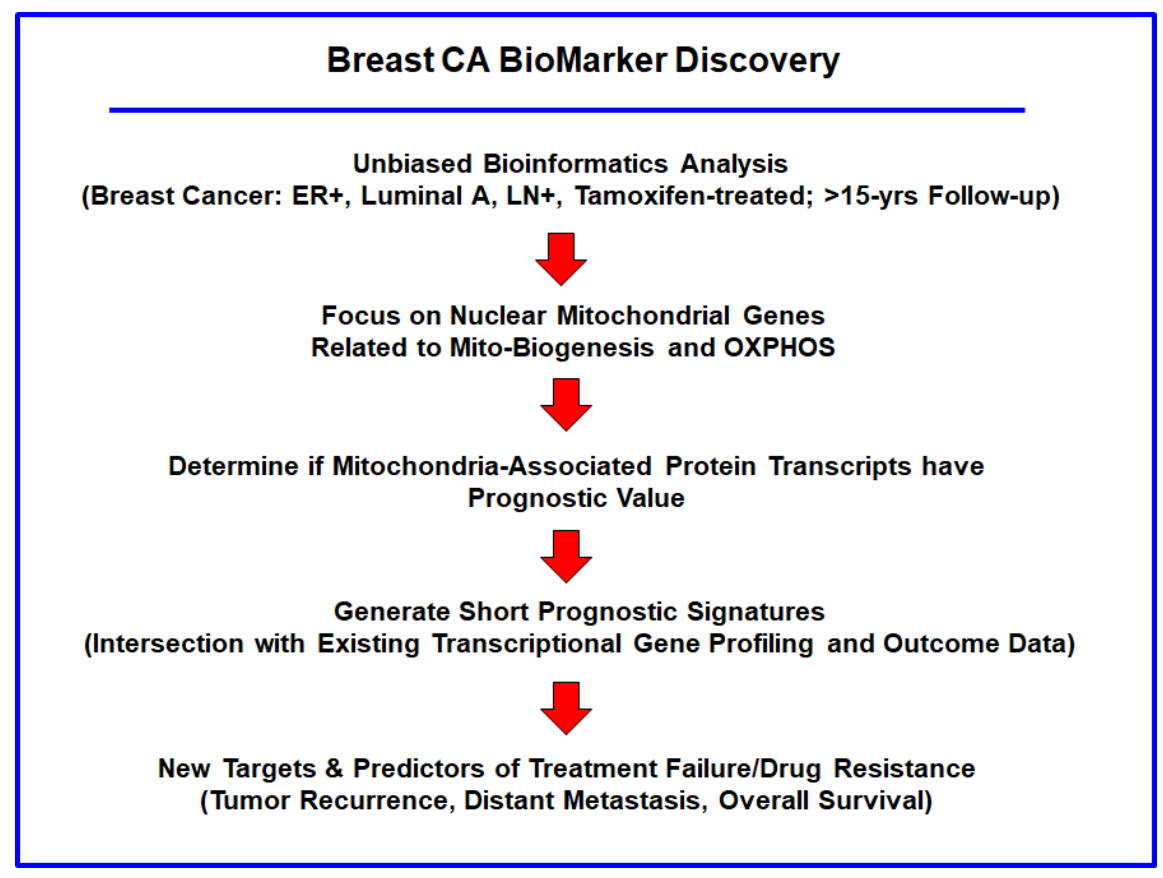

Figure 1: Flow-diagram illustrating our overall informatics approach to breast cancer biomarker discovery for predicting tamoxifen-resistance. For this analysis, we chose to focus on ER(+) patients, luminal A sub-type, that were lymph-node positive $(\mathrm{LN}(+))$ at diagnosis, who were treated with tamoxifen and followed over a period of nearly 200 months ( $>15$ years). In this context, we evaluated the prognostic value of mitochondrial markers for predicting tumor recurrence or distant metastasis (treatment failure), as well as overall survival, in this patient population. 
Table 1: Prognostic value of known markers of proliferation

\begin{tabular}{llll}
\hline Gene Probe ID & Symbol & Hazard-Ratio & Log-Rank Test \\
212022_s_at & MKI67 & 2.52 & 0.002 \\
217400_at & PCNA & 1.81 & 0.04
\end{tabular}

Table 2: Prognostic value of known markers of ER-signaling

\begin{tabular}{llll}
\hline Gene Probe ID & Symbol & Hazard-Ratio & Log-Rank Test \\
& & & \\
205225_at & ESR1 & 0.31 & 0.003 \\
208711_s_at & CCND1 & 0.53 & 0.025 \\
200952_s_at & CCND2 & 0.50 & 0.03 \\
\hline
\end{tabular}

A
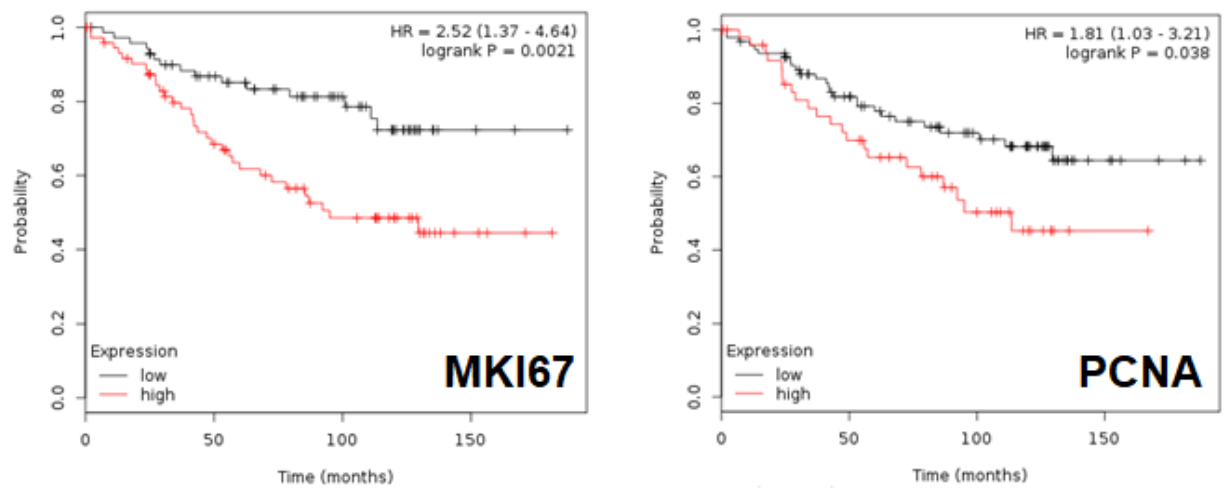

B

ER-Signaling

RFS $\mathbf{N}=145$ (Recurrence)
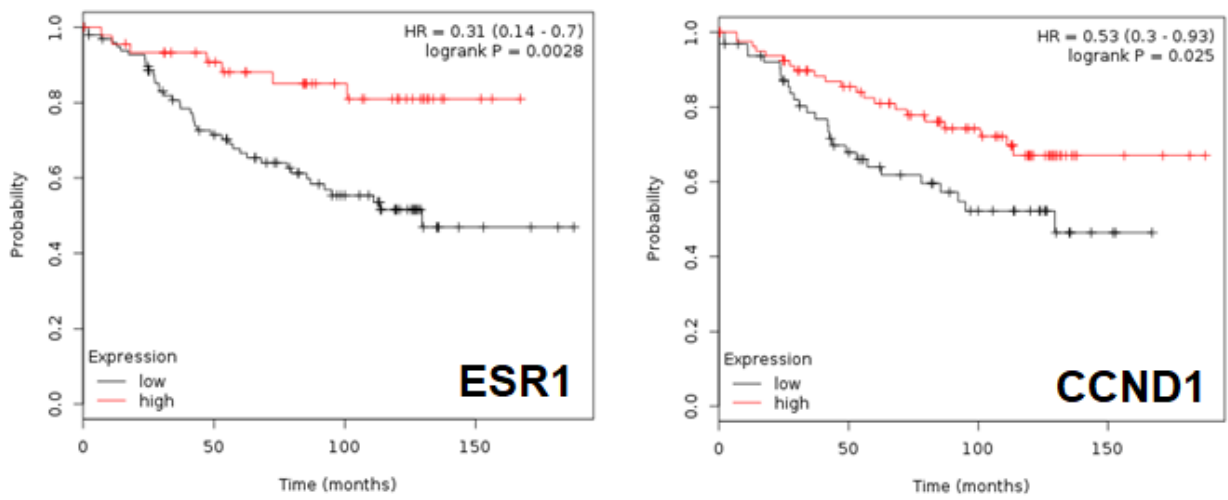

Figure 2: Conventional markers of proliferation and estrogen-receptor-alpha signaling predict clinical outcome in high-risk ER(+) breast cancer patients. We assessed the predictive value of Ki67 and PCNA in $N=145$ ER $(+)$ breast cancer patients, luminal A sub-type, that were lymph-node positive $(\mathrm{LN}(+))$ at diagnosis, who were treated with tamoxifen and followed over a period of nearly 200 months ( $>15$ years). A. Note that high transcript levels of Ki67 and PCNA are associated with increased levels of tumor recurrence, indicative of tamoxifen-resistance. Please note that the official gene name for the Ki67 protein is MKI67. B. Note that high transcript levels of estrogen-receptor (ESR1) and cyclin D1 expression (CCND1) are both associated with reduced tumor recurrence, showing increased efficacy of tamoxifen therapy. RFS, recurrence or relapse free survival is shown (a.k.a., tumor recurrence). 
Table 3: Prognostic value of markers of inflammation

\begin{tabular}{llll}
\hline Gene Probe ID & Symbol & Hazard-Ratio & Log-Rank Test \\
& & & \\
216233_at & CD163 & 2.95 & 0.02 \\
215049_x_at & CD163 & 2.45 & 0.009 \\
203645_s_at & CD163 & 2.34 & 0.003 \\
203507_at & CD68 & 1.76 & 0.048 \\
Combined & & $\mathbf{2 . 3 1}$ & $\mathbf{0 . 0 0 3}$ \\
\hline
\end{tabular}

prognostic value of these markers. The hazard-ratios for Ki67 and PCNA were 2.5 and 1.8, respectively, for relapse-free survival (RFS) (i.e., tumor recurrence).

Next, we assessed the behavior of markers of estrogen receptor signaling in these patients. It would be predicted that increased levels of such markers would be associated with a positive response to hormonal therapy. As predicted, Table 2 and Figure 2B show that estrogen receptor-alpha (ESR1) and cyclin D1/2 levels (CCND1/2) both effectively predict tamoxifen-sensitivity, as reflected by a reduction in tumor recurrence.

Finally, we also assessed the prognostic value of two macrophage-specific markers of inflammation. Table 3 and Figure 3 show that CD68 and CD163 both effectively predict tumor recurrence, with hazard-ratios of 1.76 and 2.95 , respectively.

Thus, conventional markers of proliferation, estrogen signaling, and inflammation can all be used to predict tumor-recurrence and tamoxifen-resistance in $\mathrm{LN}(+)$ luminal A breast cancer patients.

\section{Prognostic value of individual markers of mitochondrial biogenesis}

To test our hypothesis that increased mitochondrial biogenesis contributes towards tumor recurrence and tamoxifen-resistance, we next assessed the prognostic value of specific mitochondrial markers.

First, we examined the behavior of mitochondrial chaperones (HSPs) and mitochondrial membrane proteins (TIMM/TOMM/VDAC families). Table 4 and Figure 4 show that HSP60 (HSPD1) and VDAC2 have the best prognostic value with hazard-ratios of 3.6 and 4.2, respectively. Importantly, several members of the TIMM and TOMM gene families also had prognostic value $(\mathrm{HR}=$ 1.8-to-2.8). AKAP1 and IMMT also had significant value $(\mathrm{HR}=1.8$-to-2.2). Notably, the mitochondrial anti-oxidant SOD2 also showed significant prognostic value, with a hazard-ratio of $2.94(p=0.0001)$ (Table 4). Similar results were obtained with mitochondrial creatine kinase isoforms $(\mathrm{HR}=2.0$-to-2.2).

\section{Inflammation/Macrophages}
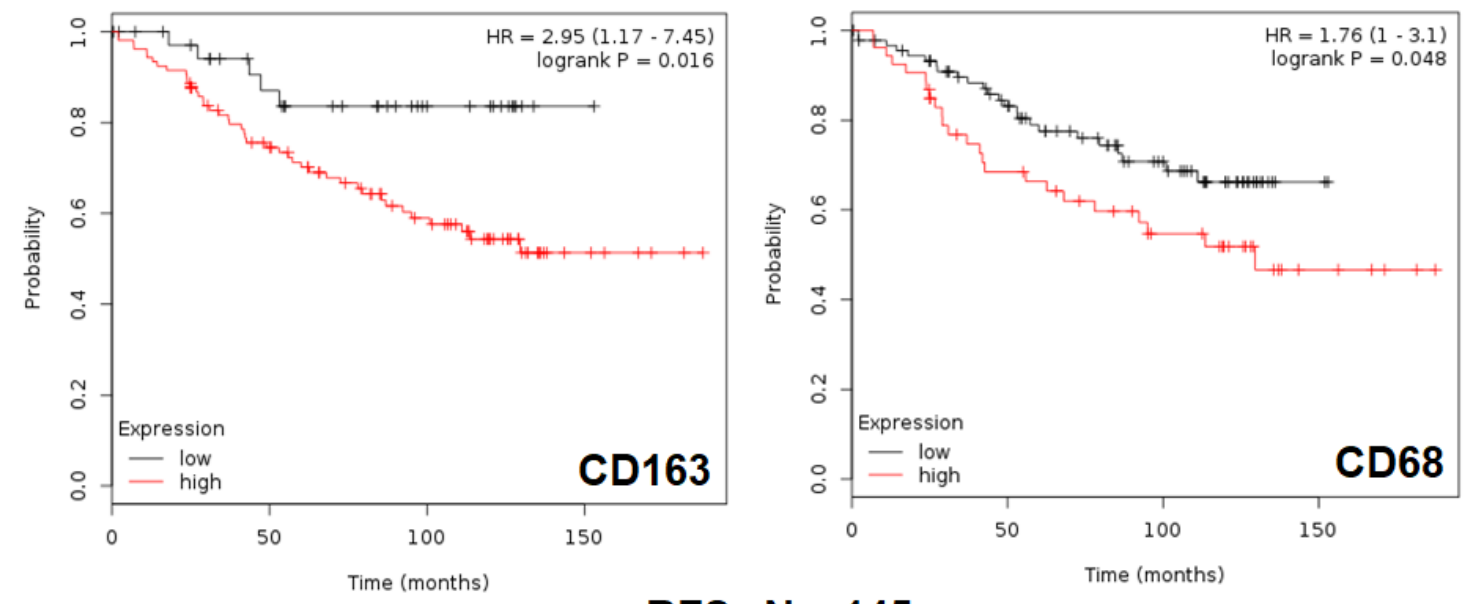

\section{RFS $\mathbf{N}=145$ \\ (Recurrence)}

Figure 3: Conventional markers of macrophage-associated inflammation predict poor clinical outcome in high-risk ER(+) breast cancer patients. Note that that high transcript levels of CD163 and CD68 are associated with increased levels of tumor recurrence and, therefore, tamoxifen-resistance. 
Table 4: Prognostic value of mitochondrial chaperones, membrane proteins, carriers, anti-oxidants and creatine kinase

\begin{tabular}{|c|c|c|c|}
\hline Gene Probe ID & Symbol & Hazard-Ratio & Log-Rank Test \\
\hline \multicolumn{4}{|c|}{ Mito Chaperones } \\
\hline 200807_s_at & HSPD1 & 3.61 & $5.9 e-06$ \\
\hline 200806 s at & HSPD1 & 2.30 & 0.006 \\
\hline 200691_s_at & HSPA9 & 2.04 & 0.01 \\
\hline 205565_s_at & FXN & 1.83 & 0.038 \\
\hline 221235_s_at & TRAP1 & 1.79 & 0.047 \\
\hline \multicolumn{4}{|c|}{ Mito Membrane Proteins } \\
\hline 211662_s_at & VDAC2 & 4.17 & $2.2 \mathrm{e}-07$ \\
\hline 210626_at & AKAP1 & 2.15 & 0.01 \\
\hline 200955_at & IMMT & 1.81 & 0.04 \\
\hline 201519_at & TOMM70A & 2.78 & 0.0003 \\
\hline 201512_s_at & TOMM70A & 2.15 & 0.01 \\
\hline 203093_s_at & TIMM44 & 2.23 & 0.01 \\
\hline 218188_s_at & TIMM13 & 2.23 & 0.02 \\
\hline 201822_at & TIMM17A & 2.01 & 0.01 \\
\hline 215171_s_at & TIMM17A & 1.85 & 0.04 \\
\hline 203342_at & TIMM17B & 1.78 & 0.04 \\
\hline \multicolumn{4}{|c|}{ Mito Carrier Family } \\
\hline 217961_at & SLC25A38 & 2.77 & 0.0003 \\
\hline 210010_s_at & SLC25A1 & 2.38 & 0.002 \\
\hline 200657_at & SLC25A5 & 2.04 & 0.01 \\
\hline 221020_s_at & SLC25A32 & 1.98 & 0.02 \\
\hline \multicolumn{4}{|c|}{ Mito Anti-Oxidants } \\
\hline 215223_s_at & SOD2 & 2.94 & 0.0001 \\
\hline 215078_at & SOD2 & 2.81 & 0.008 \\
\hline \multicolumn{4}{|c|}{ Mito Creatine Kinase } \\
\hline 205295_at & CKMT2 & 2.18 & 0.04 \\
\hline 202712_s_at & CKMT1A & 2.03 & 0.02 \\
\hline
\end{tabular}

Next, we examined the prognostic value of all the known mitochondrial ribosomal proteins (MRPs), which contribute to the protein translation of key members of the OXPHOS-related complexes, and are essential for mitochondrial biogenesis (summarized in Table 5).

Twelve different components of the large subunit (MRPLs) showed significant prognostic value, with hazard-ratios between 1.8 and 3.3. Most notably, MRPL15 had the best prognostic value ( $\mathrm{HR}=3.3 ; p=1.6 \mathrm{e}-05)$.
Similarly, six different components of the small subunit (MRPSs) showed significant prognostic value, with hazard-ratios between 1.8 and 2.35.

Thus, 18 different MRPs all predicted tumor recurrence. Kaplan-Meier curves for representative examples are shown in Figure 5, panels A \& B.

We also assessed the prognostic value of members of the OXPHOS complexes I-V. These results are summarized in Table 6. Remarkably, greater than 20 


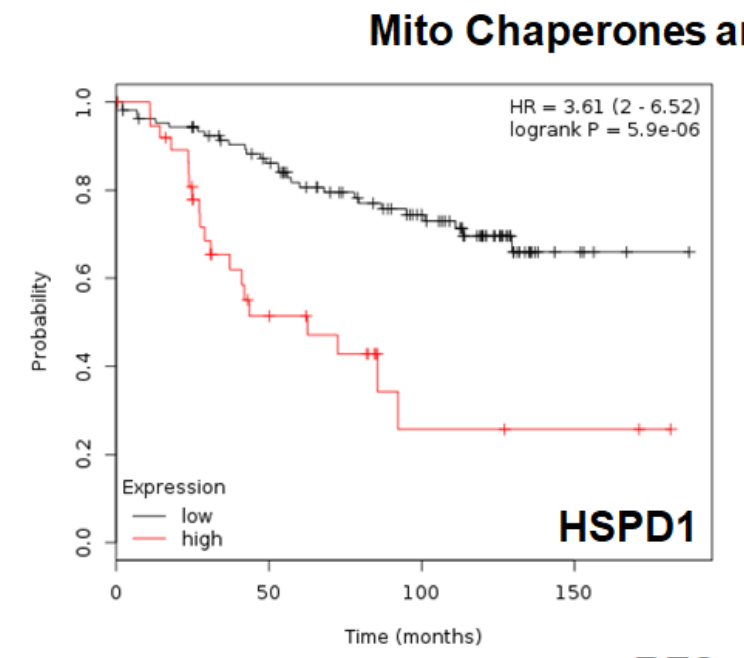

Mito Chaperones and Membrane Proteins

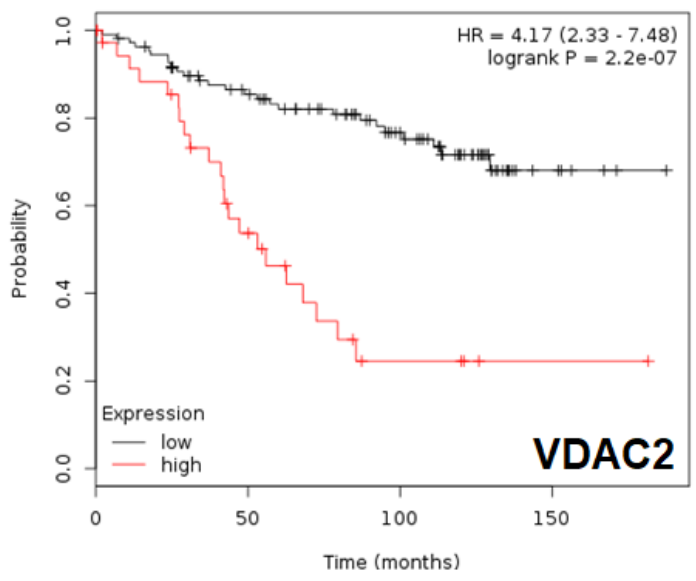

RFS $\mathbf{N}=145$

(Recurrence)

Figure 4: Mitochondrial chaperones and membrane proteins are associated with tumor recurrence in high-risk ER(+) breast cancer patients. Note that that high transcript levels of HSPD1 and VDAC2 are associated with increased levels of tumor recurrence and resistance to hormonal therapy.
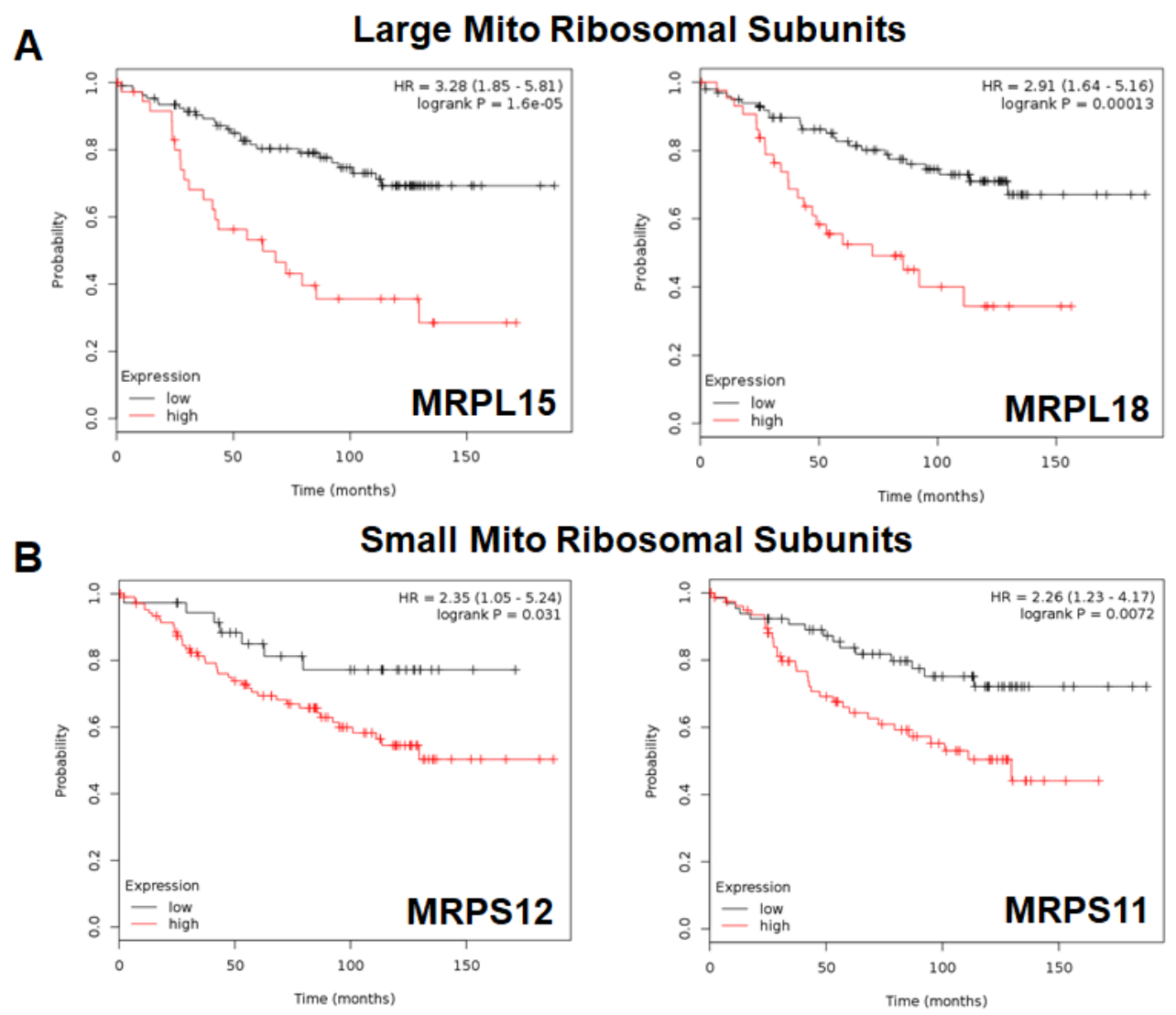

RFS $N=145$

(Recurrence)

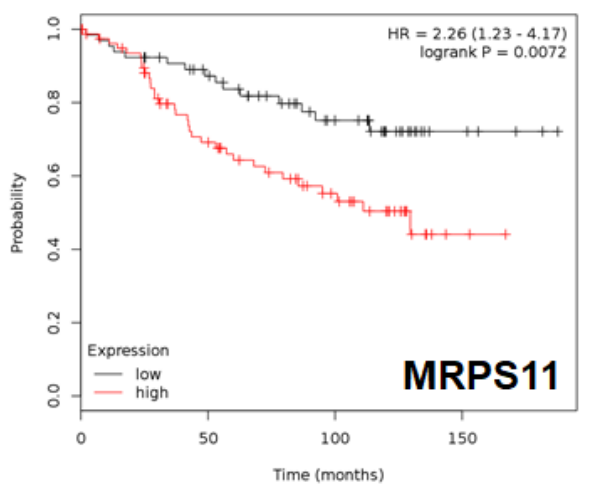

Figure 5: Mitochondrial ribosomal proteins (MRPs) are associated with tumor recurrence in high-risk ER(+) breast cancer patients. A. Note that high transcript levels of MRPL15 and MRPL18 predict increased tumor recurrence and tamoxifenresistance. B. Similarly, high transcript levels of MRPS12 and MRPS11 predict increased tumor recurrence and tamoxifen-resistance. 
A

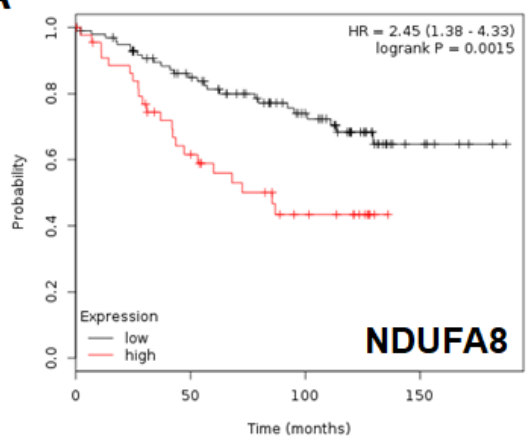

Mito Complex I

B

Mito Complex III

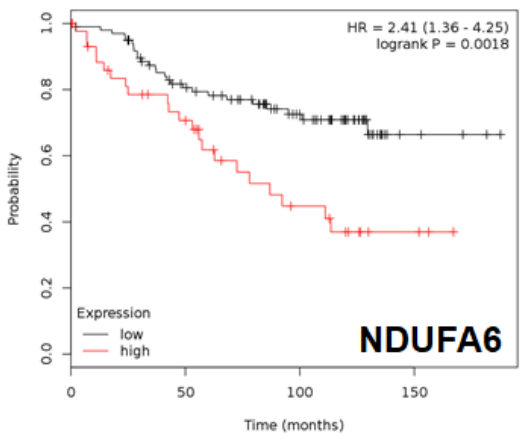

RFS $N=145$ (Recurrence)
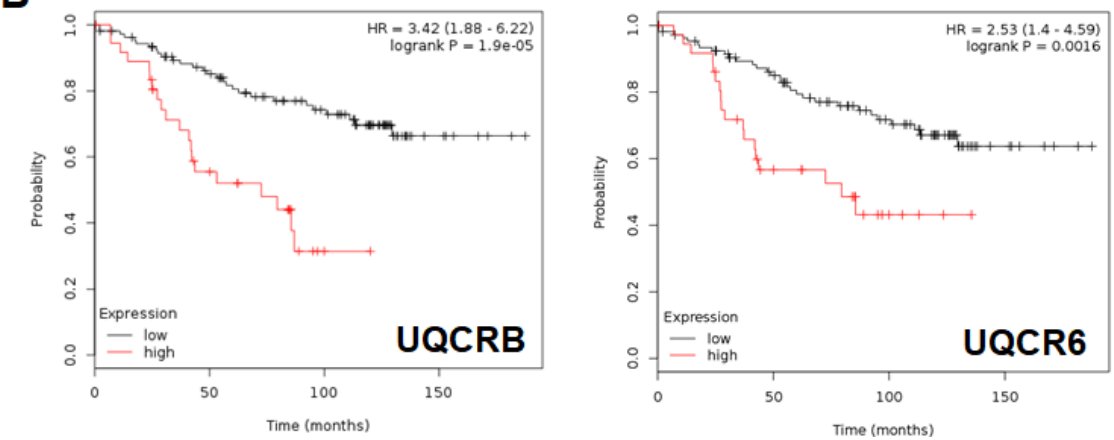

Figure 6: Mitochondrial complex I and complex III proteins are associated with tumor recurrence in high-risk ER(+) breast cancer patients. A. Note that high levels of NDUFA8 and NDUFA6 predict increased tumor recurrence and tamoxifen-resistance. B. Similarly, high levels of UQCRB and UQCR6 predict increased tumor recurrence and tamoxifen-resistance.

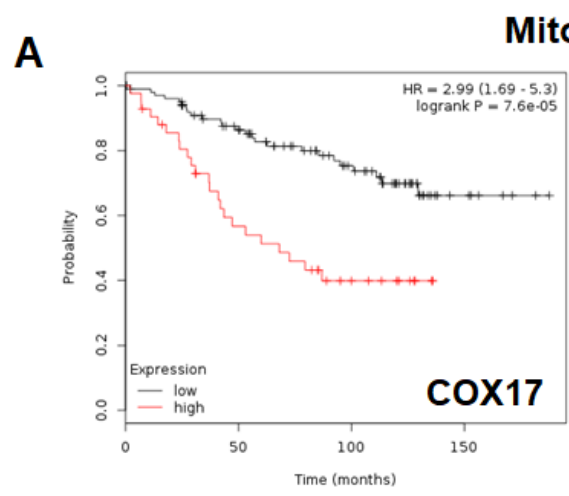

Mito Complex IV
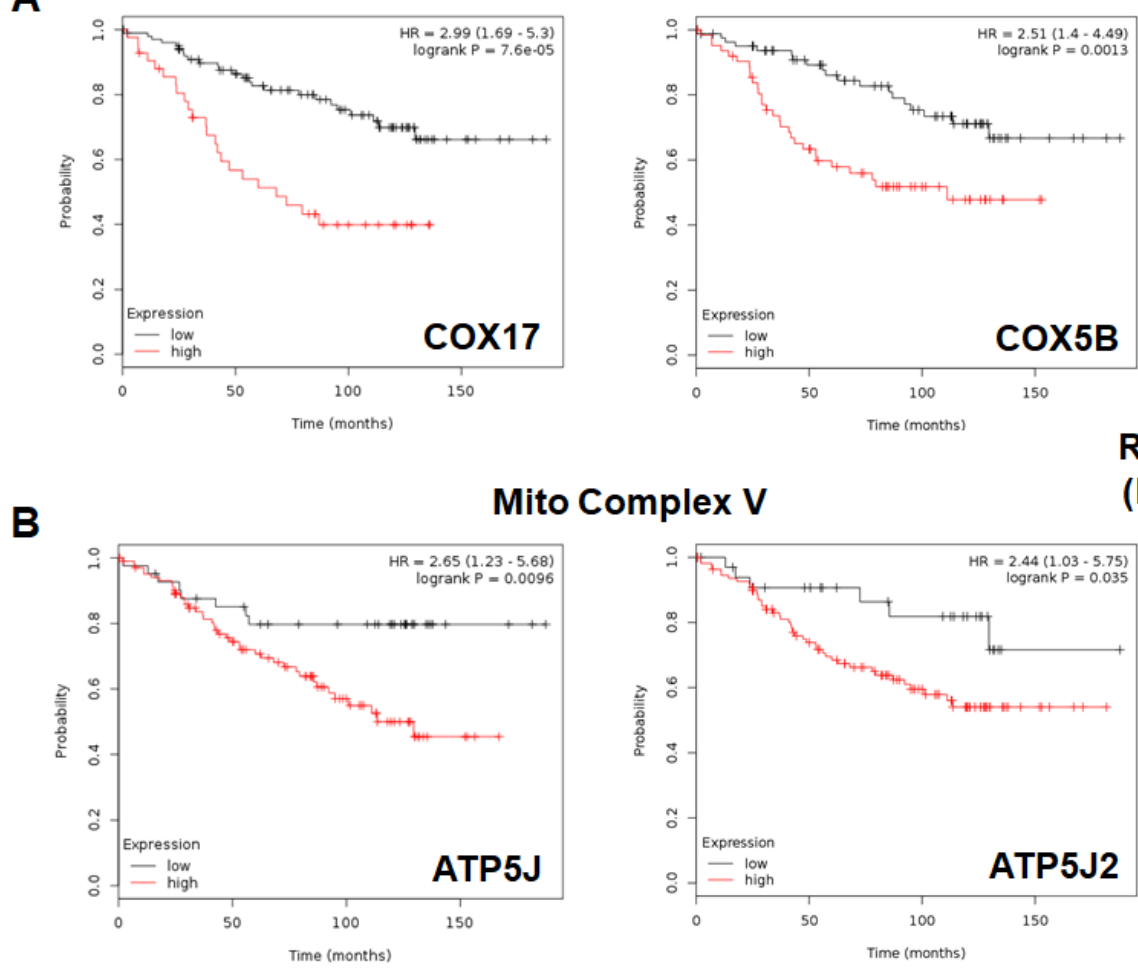

RFS $\mathrm{N}=145$

(Recurrence)

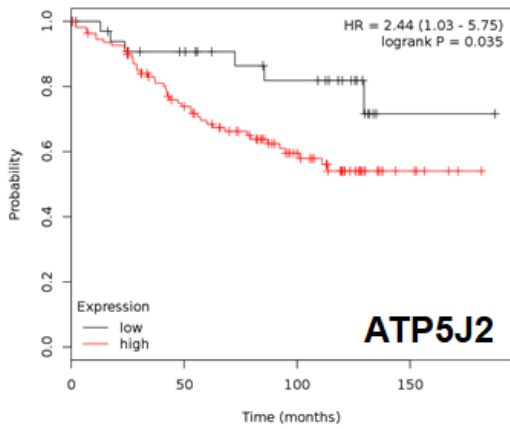

Figure 7: Mitochondrial complex IV and complex V proteins are associated with tumor recurrence in high-risk ER(+) breast cancer patients. A. Note that high levels of COX17 and COX5B predict increased tumor recurrence and tamoxifen-resistance. B. Similarly, high levels of ATP5J and ATP5J2 predict increased tumor recurrence and tamoxifen-resistance. 
Table 5: Prognostic value of mitochondrial Ribosomal proteins

\begin{tabular}{llll}
\hline Gene Probe ID & Symbol & Hazard-Ratio & Log-Rank Test \\
& & & \\
Large Ribosomal Subunit & & $1.6 e-05$ \\
218027 at & MRPL15 & 3.28 & 0.0001 \\
217907_at & MRPL18 & 2.91 & 0.02 \\
219244_s_at & MRPL46 & 2.89 & 0.002 \\
218270_at & MRPL24 & 2.38 & 0.01 \\
218049 _s_at & MRPL13 & 2.14 & 0.01 \\
218281_at & MRPL48 & 2.11 & 0.03 \\
208787_at & MRPL3 & 2.07 & 0.04 \\
213897_s_at & MRPL23 & 2.02 & 0.02 \\
218105_s_at & MRPL4 & 1.99 & 0.02 \\
222216_s_at & MRPL17 & 1.97 & 0.05 \\
217919_s_at & MRPL42 & 1.88 & 0.04 \\
218202_x_at & MRPL44 & 1.78 & \\
& & & \\
Small Ribosomal Subunit & & 0.03 \\
204330_s_at & MRPS12 & 2.35 & 0.01 \\
211595_s_at & MRPS11 & 2.26 & 0.03 \\
219819_s_at & MRPS28 & 1.88 & 0.05 \\
217919_s_at & MRPL42 & 1.88 & 0.04 \\
219220_x_at & MRPS22 & 1.85 & 0.04 \\
218654_s_at & MRPS33 & 1.84 & \\
& & & \\
\hline
\end{tabular}

\section{Mito Signature 1}

(HSPD1/UQCRB/MRPL15/COX17)

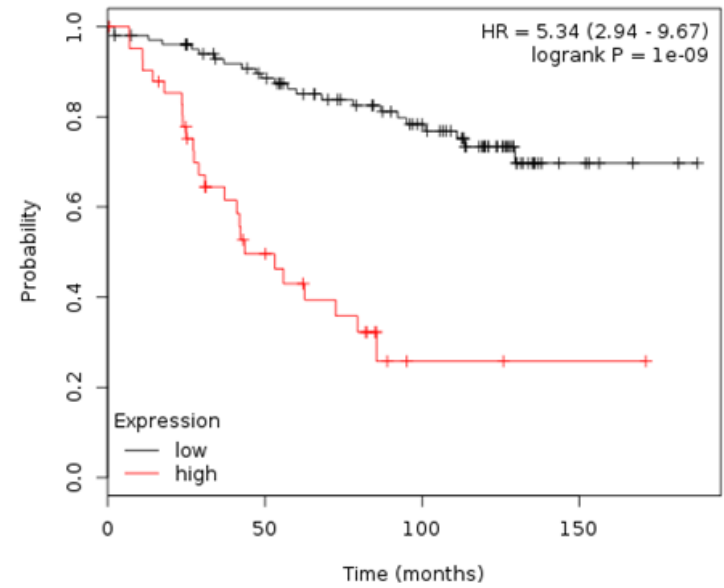

RFS $N=145$

(Recurrence)

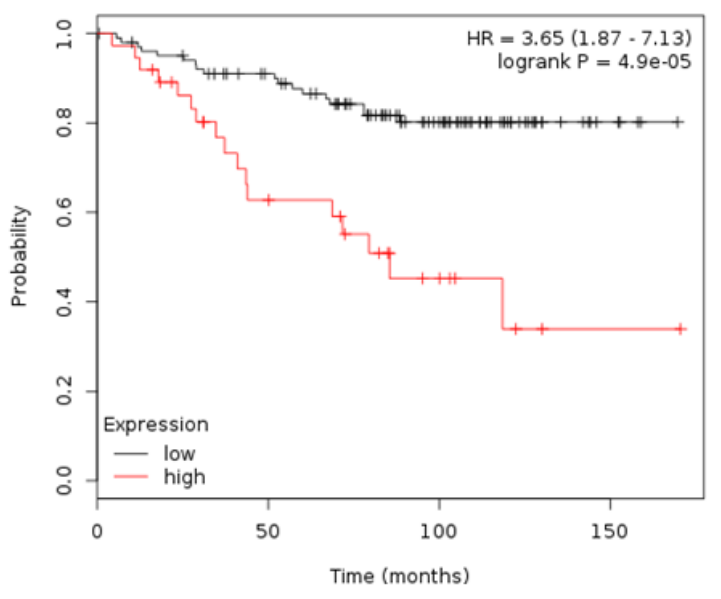

DMFS $N=139$ (Distant Metastasis)

Figure 8: A short mitochondrial signature (Mito-Signature-1) predicts poor clinical outcome in high-risk ER(+) breast cancer patients. Note that this short 4-gene signature (HSPD1/UQCRB/MRPL15/COX17) effectively predicts tumor recurrence and distant metastasis in $\mathrm{LN}(+)$ luminal A patients treated with tamoxifen therapy, indicative of treatment failure and tamoxifen-resistance. 
Table 6: Prognostic value of mitochondrial OXPHOS complexes

Gene Probe ID Symbol Hazard-Ratio Log-Rank Test

\section{Complex I}

218160_at

202000_at

202001_s_at

203039_s_at

201740_at

203613_s_at

208714_at

203606_at

202298_at

Complex III

209065_at

209066_x_at

205849_s_at

201066_at

212600_s_at

\section{Complex IV}

203880_at

213735_s_at

202343_x_at

211025_x_at

202698_x_at

200925_at

218057_x_at

217249_x_at

Complex V

202325_s_at

202961_s_at

213366_x_at

208870_x_at

205711_x_at

217848_s_at

221677_s_at

217801_at

207508_at
NDUFA8

NDUFA6

NDUFA6

NDUFS1

NDUFS3

NDUFB6

NDUFV1

NDUFS6

NDUFA1

UQCRB

UQCRB

UQCR6

UQCR4

UQCRC2

CoX17

COX5B

COX5B

COX5B

COX4I1

COX6A1

COX4NB

COX7A2

ATP5J

ATP5J2

ATP5C1

ATP5C1

ATP5C1

PPA1

ATP5O

ATP5E

ATP5G3

\subsection{5}

2.41

2.23

2.40

2.17

1.99

1.96

1.92

1.89

3.42

2.12

2.53

1.96

1.92

2.99

2.51

2.10

2.08

2.36

2.14

1.99

1.90

2.65

2.44

2.19

2.08

2.00

2.07

2.03

1.99

1.93
0.002

0.002

0.006

0.003

0.006

0.02

0.03

0.04

0.03

$1.9 \mathrm{e}-05$

0.01

0.002

0.02

0.04
7.6e-05
0.001
0.01
0.01
0.02
0.01
0.04
0.03

0.01

0.035

0.01

0.01

0.02

0.01

0.02

0.02

0.02

Table 7: Mito-signature 1 for predicting treatment failure

\begin{tabular}{llll}
\hline Gene Probe ID & Symbol & Hazard-Ratio & Log-Rank Test \\
& & & \\
200807_s_at & HSPD1 & 3.61 & $5.9 \mathrm{e}-06$ \\
209065_at & UQCRB & 3.42 & $1.9 \mathrm{e}-05$ \\
218027_at & MRPL15 & 3.28 & $1.6 \mathrm{e}-05$ \\
203880_at & COX17 & 2.99 & $7.6 \mathrm{e}-05$ \\
Combined & & $\mathbf{5 . 3 4}$ & $\mathbf{1 e - 0 9}$ \\
\hline
\end{tabular}


Table 8: Mito-signature 2 for predicting treatment failure

\begin{tabular}{llll}
\hline Gene Probe ID & Symbol & Hazard-Ratio & Log-Rank Test \\
& & & \\
211662_s_at & VDAC2 & 4.17 & $2.2 \mathrm{e}-07$ \\
200807_s_at & HSPD1 & 3.61 & $5.9 \mathrm{e}-06$ \\
Combined & & $\mathbf{5 . 1 9}$ & $\mathbf{6 e - 0 9}$
\end{tabular}

different members of the OXPHOS complexes showed hazard-ratios between 1.9 and 3.4. UQCRB (complex III) had the best prognostic value (HR $=3.42 ; p=1.9 \mathrm{e}-$ 05). Similarly, COX17 (complex IV) showed significant prognostic value $(\mathrm{HR}=2.99 ; p=7.6 \mathrm{e}-05)$.

Kaplan-Meier curves for members of complex I and III are shown in Figure 6A \& B, while results with members of complex IV and $\mathrm{V}$ are also shown in Figure $7 \mathrm{~A} \& 7 \mathrm{~B}$.

Two new mitochondrial gene signatures for predicting tumor recurrence, distant metastasis and tamoxifen-resistance

In order to increase the prognostic power of these individual mitochondrial biomarkers, we next selected the most promising ones and used them to create two new mitochondrial gene signatures. Mito-Signature-1 contains 4 genes (HSPD1, UQCRB, MRPL15, COX17), while Mito-Signature- 2 consists of only 2 genes (HSPD1, VDAC2) (See Tables $7 \&$ 8). K-M curves for these two signatures are shown in Figures 8 and 9.

Importantly, Mito-Signature-1 yielded a significantly improved hazard-ratio for tumor recurrence of 5.34 ( $p=$ 1e-09). It was also highly predictive for distant metastasis, in the same group of patients ( $\mathrm{HR}=3.65 ; p=4.9 \mathrm{e}-05)$.

Similarly, Mito-Signature-2 showed a hazard-ratio for tumor recurrence of $5.2(p=6 \mathrm{e}-09)$. Mito-Signature-2 was also highly predictive for distant metastasis $(\mathrm{HR}=$ $3.88 ; p=6.8 \mathrm{e}-05$ ).

Thus, both mitochondrial signatures were a significant improvement over individual mitochondrial biomarkers, as well as Ki67, PCNA, ESR1, CCND1/2 and CD68/CD163 (compare with Figures $2 \& 3$ ).

\section{Mito Signature 2}
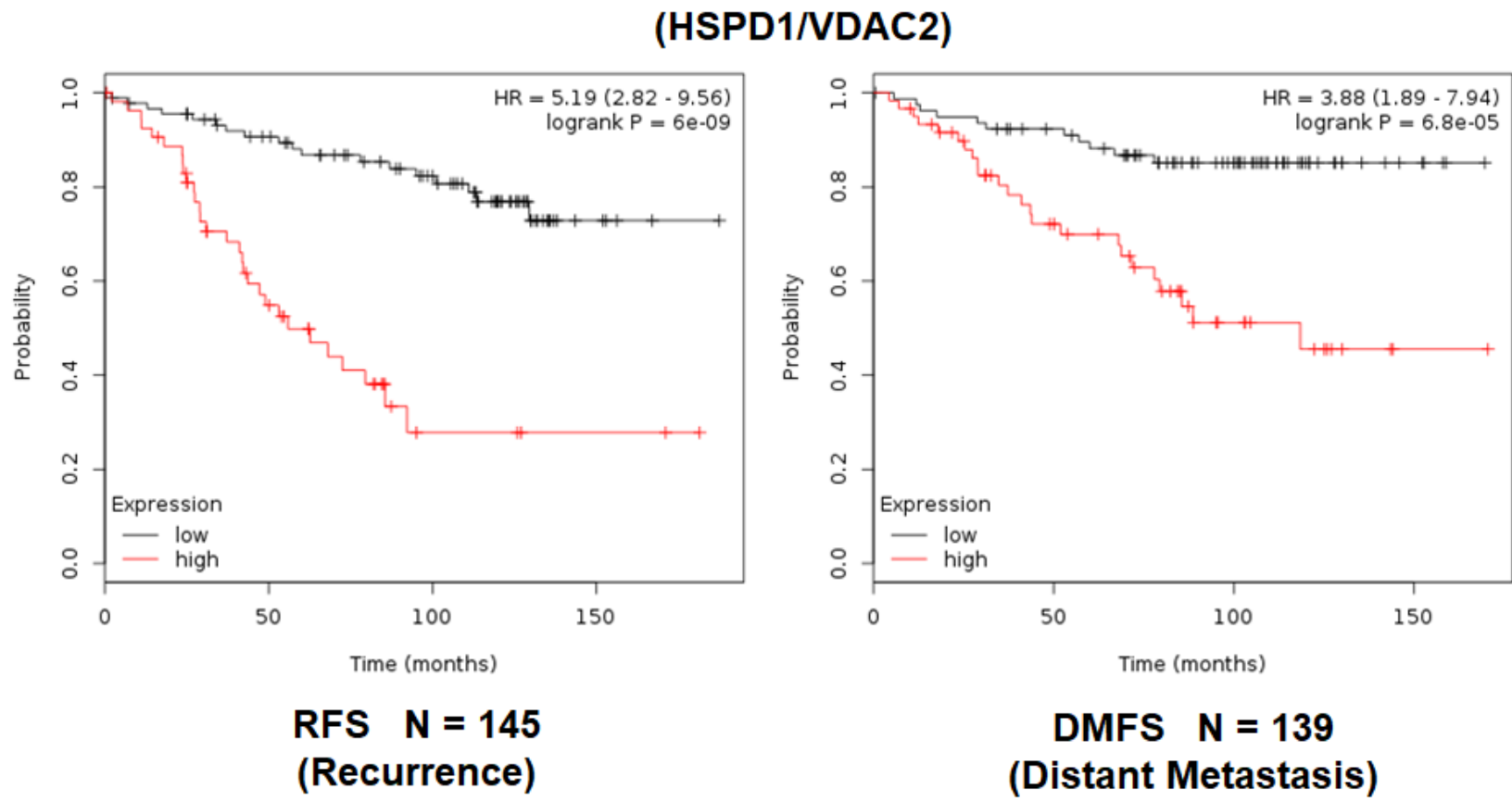

Figure 9: A short mitochondrial signature (Mito-Signature-2) predicts poor clinical outcome in high-risk ER(+) breast cancer patients. Note that this short 2-gene signature (HSPD1/VDAC2) effectively predicts tumor recurrence and distant metastasis in $\mathrm{LN}(+)$ luminal A patients treated with tamoxifen therapy, indicative of treatment failure and tamoxifen-resistance. 


\section{All ER+ BrCa Treated with Hormonal Therapy}

A

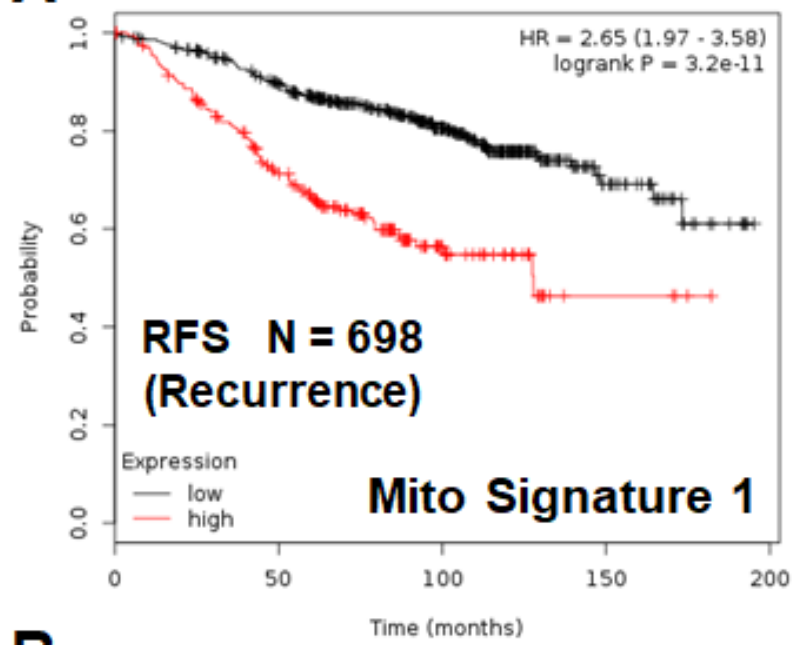

B

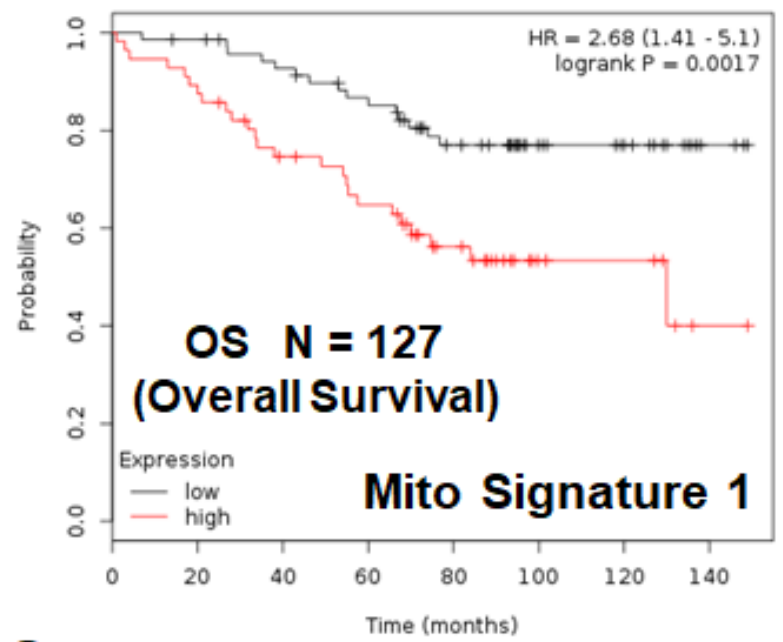

C

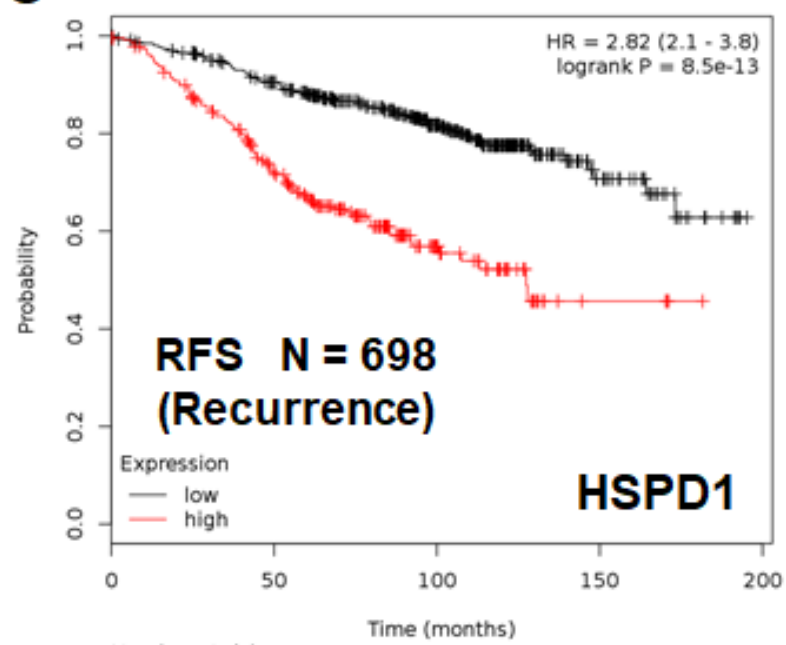

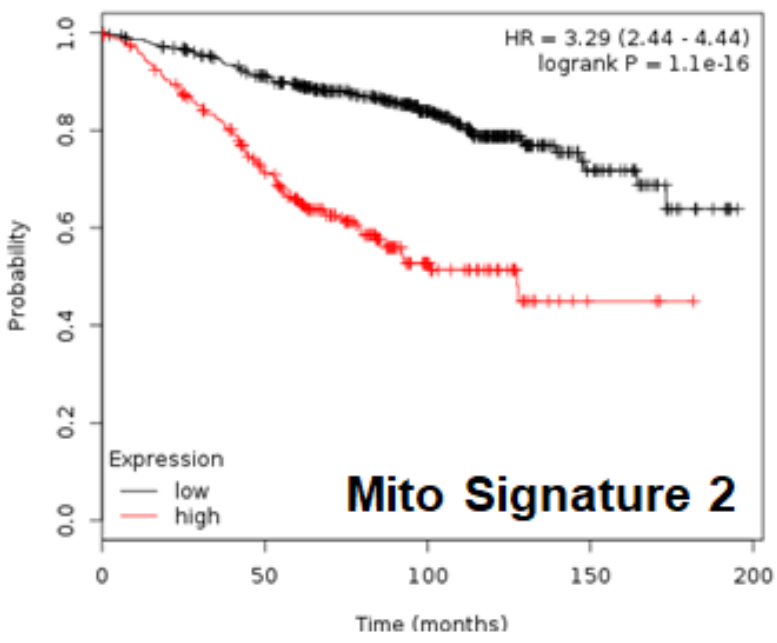
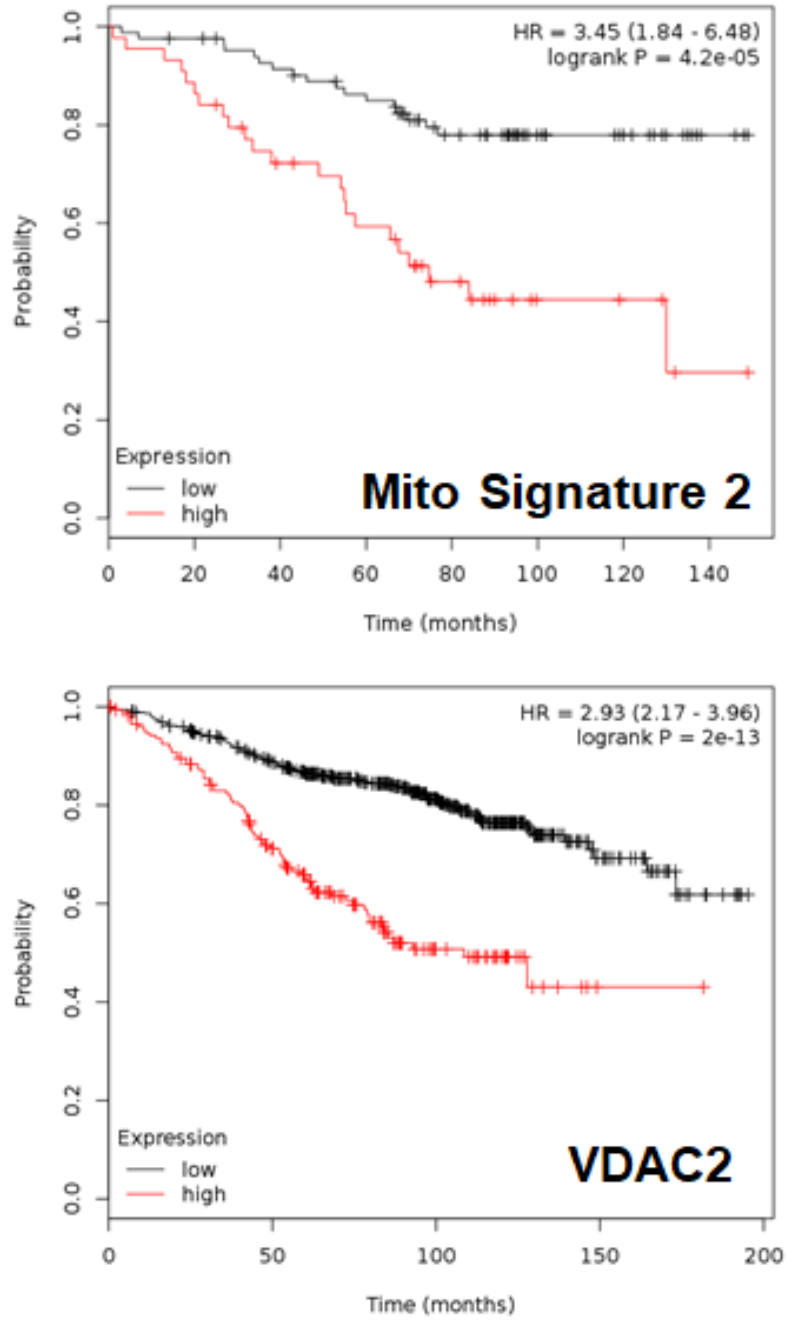

Figure 10: Mitochondrial signatures 1 and 2 both have predictive value in a larger group of ER(+) breast cancer patients, who were treated with hormonal therapy. These patients were not sub-divided into luminal A/B subgroups and were not sub-divided by lymph-node status. A. K-M analysis with Mito-Signatures $1 \& 2$, showing tumor recurrence. $N=698$ patients. B. K-M analysis with Mito-Signatures $1 \&$ 2, showing overall survival. $N=127$ patients. C. K-M analysis with individual markers (HSPD1 and VDAC2) is also shown for comparison. $N=698$ patients. 
All ER+ BrCa Treated with Hormonal Therapy
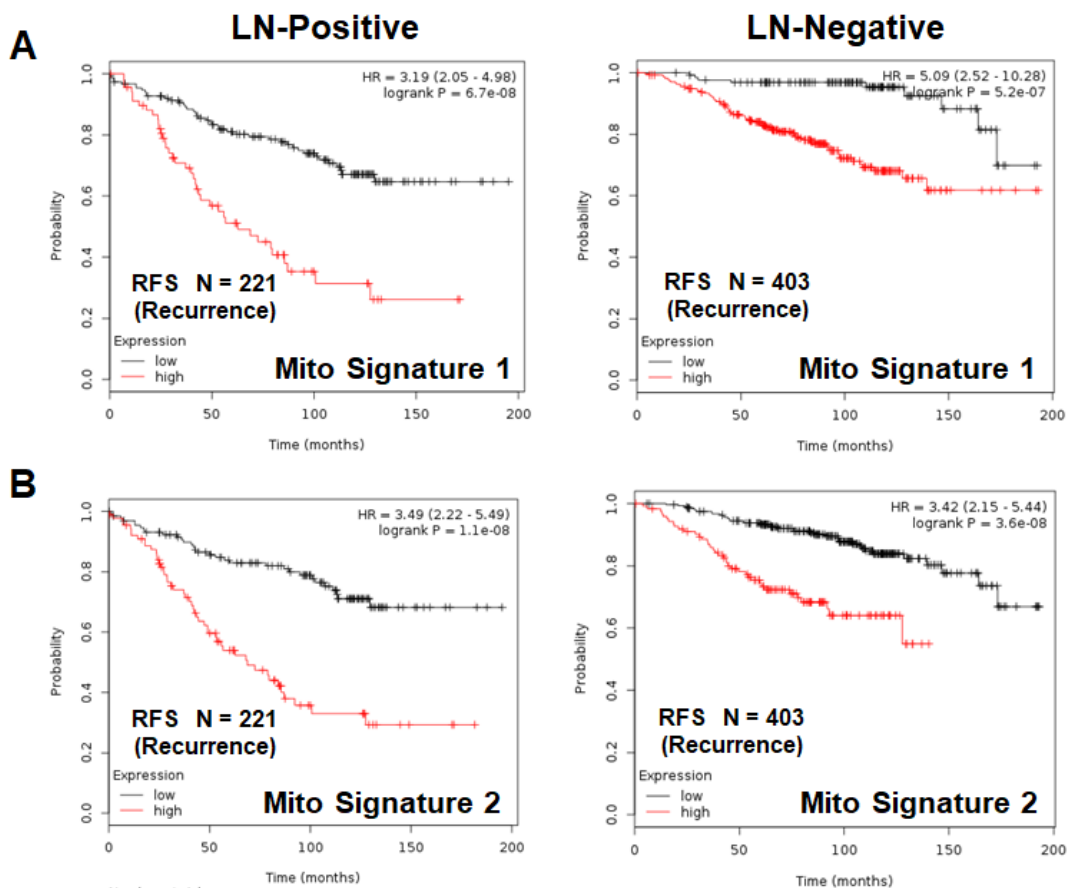

Figure 11: Mitochondrial signatures 1 and 2 both have predictive value in a larger group of ER(+) breast cancer patients, who were treated with hormonal therapy. These patients were not sub-divided into luminal A/B subgroups, but were sub-divided by lymph-node status $(\mathrm{LN}(+)$ versus $\mathrm{LN}(-))$. A. K-M analysis with Mito-Signature-1 is shown for both groups: $\operatorname{LN}(+)(N=$ 221 patients) and $\mathrm{LN}(-)(N=403$ patients). B. K-M analysis with Mito-Signature- 2 is shown for both groups: $\mathrm{LN}(+)(N=221$ patients) and $\mathrm{LN}(-)(N=403$ patients $)$.

\section{All ER+ BrCa Treated with Hormonal Therapy}
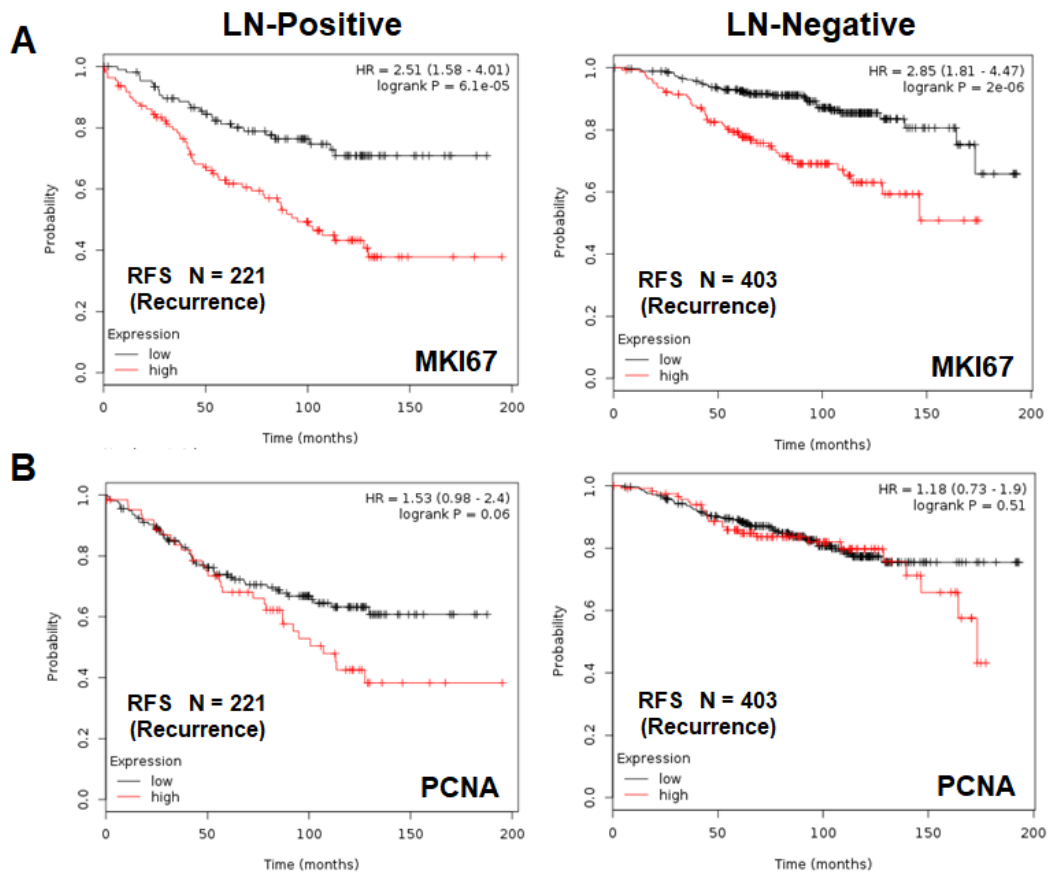

Figure 12: K-M analysis with conventional proliferative markers, in the same patient population, is shown for comparison. Note that Mito Signature $1 \& 2$ show better predictive value than both proliferative markers, namely KI67 and PCNA. A. K-M analysis with KI67 is shown for both groups: $\mathrm{LN}(+)(N=221$ patients) and $\mathrm{LN}(-)(N=403$ patients). B. K-M analysis with PCNA is shown for both groups: $\mathrm{LN}(+)(N=221$ patients $)$ and $\mathrm{LN}(-)(N=403$ patients $)$. 
Two short mitochondrial gene signatures can effectively predict tumor recurrence in larger ER(+) patient populations that received hormonal therapy, as well as in ER(-) patients, and all breast cancers, considered as a single group

We also examined the prognostic value of these two mitochondrial gene signatures in a larger group of $\mathrm{ER}(+)$ patients $(N=698)$, that received hormonal therapy, but not chemotherapy. This group of patients was not segregated into luminal A and luminal B subtypes.

Figure 10A shows the results of this K-M analysis for relapse-free survival: Mito-Signature-1 $(\mathrm{HR}=2.65$; $p=3.2 \mathrm{e}-11)$ and Mitosignature-2 ( $\mathrm{HR}=3.3 ; p=1.1 \mathrm{e}-$ 16). Similar results were also obtained for overall survival (Figure 10B).

Both of these mitochondrial signatures were also effective if the $\mathrm{ER}(+)$ patient population was divided into $\mathrm{LN}(+)$ and $\mathrm{LN}(-)$ groups (Figure 11A \& 11B). In addition, both of these mitochondrial signatures were clearly superior to Ki67 and PCNA in this larger ER(+) patient population. However, Ki67 still showed prognostic value (Figure 12A), while PCNA had no prognostic value (Figure 12B).

Finally, we assessed the behavior of MitoSignature-1 in even larger and more varied patient populations, where the therapy was not restricted to tamoxifen.

Supplementary Figure 1 shows that MitoSignature-1 was also effective in $\mathrm{ER}(+)(N=2,447)$, ER-/ basal $(N=540)$, ER-/HER2 $(+)(N=193)$, as well as in all breast cancer subtypes combined $(N=3,180)$. Similarly, Mito-Signature-1 was still statistically effective in both luminal A $(N=438+813)$ and luminal B $(N=907)$ patient populations (Supplementary Figure 2). Similarly, comparable results were obtained with Mito-Signature-2 (data not shown).

Thus, these mitochondrial-based gene signatures may represent important new prognostic tools for predicting patient outcomes, in a wide variety of different breast cancer patients, but especially in $\operatorname{ER}(+)$ patients treated with hormonal therapies.

\section{DISCUSSION}

\section{Early detection of tamoxifen-resistance with mitochondrial markers: prevention of tumor recurrence and distant metastasis?}

Here, we show that mitochondrial markers effectively predict tumor recurrence, distant metastasis and tamoxifen-resistance in high-risk $\mathrm{ER}(+)$ breast cancer patients. Importantly, these mitochondrial markers could now be used to identify high-risk ER(+) breast cancer patients at diagnosis, up to 15 years in advance, before they undergo tumor recurrence and metastasis. These results also suggest that mitochondria should be therapeutically-targeted in epithelial cancer cells to overcome tamoxifen-resistance and prevent the failure of hormonal therapy.

Consistent with this hypothesis, we have previously shown that treatment with metformin (a mitochondrial complex I inhibitor) is indeed sufficient to reverse tamoxifen-resistance in fibroblast-MCF7 co-cultures $[10,11]$. Thus, targeting mitochondrial biogenesis and OXPHOS in ER(+) epithelial breast cancer cells may be a new therapeutic strategy for preventing or reversing tamoxifen-resistance in breast cancer patients.

Interestingly, these mitochondrial markers also showed predictive value in ER(-) breast cancer patients, both basal and HER2(+), suggesting that antimitochondrial therapy could be used as a more general anti-cancer strategy, against several different breast cancer sub-types.

A schematic diagram summarizing this new mitobased approach is presented in Figure 13. In this workflow, high-risk patients are first identified at diagnosis by the high expression of mitochondrial markers in their primary breast tumors. Then, these patients would be treated with mitochondrial-based therapeutics (e.g., metformin or another FDA-approved drug; in combination with the standard of care), to help prevent tumor recurrence and distant metastasis. Alternatively, novel mitochondrial-

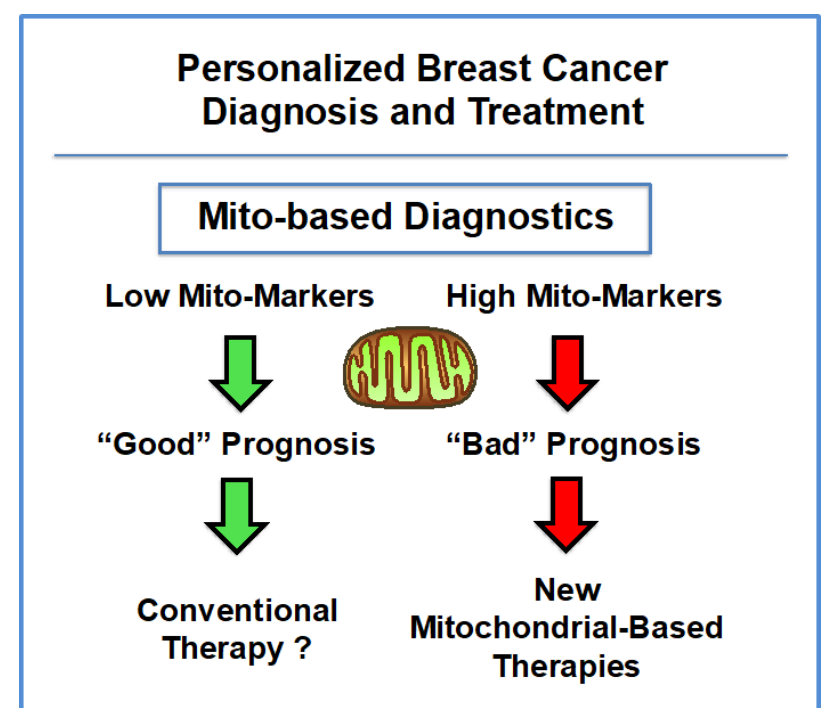

Figure 13: Mitochondrial-based companion diagnostics for personalized cancer therapy. In this flow-diagram, mitochondrial-based diagnostics would be used to separate breast cancer patients into high-risk and low-risk groups. Then, patients with high levels of mitochondrial markers in their primary tumor ("bad prognosis") would be treated with mitochondrial-based therapies (such as Metformin), as an addon to the standard of care, to prevent tumor recurrence, distant metastasis and tamoxifen-resistance. 
based chemo-therapeutics could be developed against a variety of metabolic enzymes or structural proteins, to specifically target aggressive cancer cells with increased mitochondrial function.

\section{Evidence that mitochondrial power drives tamoxifen-resistance and cancer stem cell propagation}

Consistent with the above hypothesis, we recently showed that tamoxifen-resistant MCF7 cells (TAMR) are characterized by a metabolic phenotype, consisting of i) increased mitochondrial biogenesis, ii) increased ATP production and iii) reduced glutathione levels [13]. Thus, inhibition of mitochondrial function may be a new therapeutic strategy for overcoming tamoxifenresistance in breast cancer patients. These findings could have important translational significance for the prevention of tumor recurrence in $\operatorname{ER}(+)$ breast cancers, which is due to an endocrine resistance phenotype [13]. Importantly, mitochondrial proteins may represent i) new prognostic biomarkers, ii) novel therapeutic targets and iii) companion diagnostics, for predicting and overcoming tamoxifen-resistance in different subsets of $\mathrm{ER}(+)$ breast cancer patients.

Similarly, based on high-resolution proteomics analysis, we have also proposed that mitochondrial biogenesis is an important driver of the cancer stem cell (CSC) phenotype $[14,15]$. A key correlate of this assertion is that high mitochondrial mass is a metabolic biomarker for CSCs. To directly test this idea experimentally, we used a fluorescent dye, known as MitoTracker, to detect and quantitate mitochondrial mass in $\mathrm{ER}(+)$ breast cancer cells (MCF7) [16]. Using this approach, we purified the Mitohigh and the Mito-low cell populations by flow cytometry (FACS). Remarkably, the Mito-high cell population was clearly enriched in cells with the characteristics of CSCs. Virtually identical results were also obtained with MDA-MB-231 cells, an ER(-) cell line. Thus, the use of "metabolic fractionation", employing mitochondrial-based probes and flow cytometry, could be a successful new approach to the functional purification of drug-resistant CSCs. In accordance with this hypothesis, Mito-high breast cancer cells were also resistant to DNA-damage induced by Paclitaxel [16]. Thus, mitochondrial mass and function are directly linked to i) the CSC phenotype and ii) chemotherapeutic drug resistance, as well as iii) resistance to anti-estrogen therapy [13-24]. As such, we conclude that the association we observed here of high levels of mitochondrial markers (mRNA species and/or protein products) with poor clinical outcome in breast cancer patients may functionally reflect the presence of drug-resistant CSCs, driving tumor recurrence, metastasis and treatment failure.

\section{Using mitochondrial markers as companion diagnostics for drug re-purposing, treatment stratification and new drug discovery}

Several classes of FDA-approved antibiotics safely inhibit either mitochondrial biogenesis or OXPHOS as off-target "side-effects". These include the tetracyclines (doxycycline), the erythromycins (azithromycin), pyrvinium pamoate, atovaquone, and bedaquiline, among others [13-24]. Therefore, the new mitochondrial biomarkers that we identified here could be used in combination with these FDA-approved drugs, as companion diagnostics. This would allow clinicians to select the right patient populations for new clinical trials aimed at drug re-purposing/re-positioning, for the prevention of tumor recurrence in $\mathrm{ER}(+)$ patients receiving anti-endocrine therapy.

Importantly, the novel mitochondrial biomarkers that we identified here may also be new therapeutic targets for future drug development aimed at combating the emergence of resistance to hormonal therapy. Based on our K-M analysis, the mitochondrial ribosome (a.k.a., mitoribosome) and its individual subunits would be attractive targets for intervention; in addition, mitochondrial chaperones, the OXPHOS complexes (IIV) and the mitochondrial ATP-synthase (complex V) may also be tractable targets. Since several members of each of these multi-subunit complexes show prognostic value, this provides an indication that inactivation, or specific modulation, of the activity of each of these complexes may provide significant therapeutic benefits. Therapeutic targeting of these complexes would be expected to prevent tumor recurrence and distant metastasis, as well as confer tamoxifen-sensitivity, in $\mathrm{ER}(+)$ breast cancer patients.

\section{MATERIALS AND METHODS}

\section{Kaplan-Meier (K-M) analyses}

To perform K-M analysis on $>400$ nuclear mitochondrial gene transcripts, we used an open-access online survival analysis tool to interrogate publically available microarray data from up to 3,455 breast cancer patients [12]. This allowed us to determine their prognostic value. For this purpose, we primarily analyzed data from $\mathrm{ER}(+)$ patients that were $\mathrm{LN}(+)$ at diagnosis and were of the luminal A sub-type, that were primarily treated with tamoxifen and not other chemotherapy $(N=$ 145 patients). In this group, $100 \%$ the patients received some form of hormonal therapy and $\sim 95 \%$ of them received tamoxifen. Biased and outlier array data were excluded from the analysis. This allowed us to identify $>$ 60 nuclear mitochondrial gene transcripts, with significant prognostic value. Hazard-ratios were calculated, at the best 
auto-selected cut-off, and p-values were calculated using the logrank test and plotted in R. K-M curves were also generated online using the K-M-plotter (as high-resolution TIFF files), using univariate analysis:

$\mathrm{http} / / \mathrm{kmplot} \cdot \mathrm{com} /$ analysis/index.php?p= service\&cancer $=$ breast

This allowed us to directly perform in silico validation of these mitochondrial biomarker candidates. The multi-gene classifier function of the program was used to test the prognostic value of short mitochondrial gene signatures, using the mean expression of the selected probes. The 2012 version of the database was originally utilized for all these analyses, because a higher percentage of the patients used tamoxifen; however, virtually identical results were also obtained with the 2014 and 2017 versions.

\section{Abbreviations}

CSCs, cancer stem-like cells; DMFS, distant metastasis-free survival; ER, estrogen receptor alpha (ESR1); HR, hazard ratio; K-M, Kaplan-Meier; LN, lymph node; MRPL, mitochondrial ribosomal proteins, large subunit; MRPS, mitochondrial ribosomal proteins, small subunit; $\mathrm{N}$, number of patients in a given data set; OXPHOS, oxidative phosphorylation (mitochondrial respiration); RFS, recurrence- or relapse-free survival

\section{Author contributions}

Professor Lisanti and Dr. Sotgia conceived and initiated this project. Professor Lisanti and Dr. Sotgia performed the bioinformatics analysis, and wrote the first draft of the manuscript, which was then further edited by Marco Fiorillo.

\section{ACKNOWLEDGMENTS}

It should be noted that this bioinformatics analysis, focused on nuclear-encoded mitochondrial-related gene transcripts, was not funded by a specific grant and did not require any research expenditures, since no "wet" laboratory experiments were performed.

\section{CONFLICTS OF INTEREST}

MPL and FS hold a minority interest in Lunella, Inc.

\section{REFERENCES}

1. Osborne CK, Fuqua SA. Mechanisms of tamoxifen resistance. Breast Cancer Res Treat. 1994; 32:49-55.

2. Ring A. Dowsett M. Mechanisms of tamoxifen resistance. Endocr Relat Cancer. 2004; 11:643-58.
3. Ali S, Coombes RC. Endocrine-responsive breast cancer and strategies for combating resistance. Nat Rev Cancer. 2002; 2:101-12.

4. Jordan VC. Selective estrogen receptor modulation: concept and consequences in cancer. Cancer Cell. 2004; 5:207-13.

5. Li X, Lewis MT, Huang J, Gutierrez C, Osborne CK, Wu MF, Hilsenbeck SG, Pavlick A, Zhang X, Chamness GC, Wong H, Rosen J, Chang JC. Intrinsic resistance of tumorigenic breast cancer cells to chemotherapy. J Natl Cancer Inst. 2008; 100:672-79.

6. Giuliano M, Schifp R, Osborne CK, Trivedi MV. Biological mechanisms and clinical implications of endocrine resistance in breast cancer. Breast. 2011; 20:S42-49.

7. Osborne CK, Schiff R. Mechanisms of endocrine resistance in breast cancer. Annu Rev Med. 2011; 62:233-47.

8. Clarke R, Tyson JJ, Dixon JM. Endocrine resistance in breast cancer-An overview and update. Mol Cell Endocrinol. 2015; 418:220-34.

9. Dittmer J, Leyh B. The impact of tumor stroma on drug response in breast cancer. Semin Cancer Biol. 2015; 31:315.

10. Martinez-Outschoorn UE, Goldberg A, Lin Z, Ko YH, Flomenberg N, Wang C, Pavlides S, Pestell RG, Howell A, Sotgia F, Lisanti MP. Anti-estrogen resistance in breast cancer is induced by the tumor microenvironment and can be overcome by inhibiting mitochondrial function in epithelial cancer cells. Cancer Biol Ther. 2011; 12:924-38.

11. Martinez-Outschoorn UE, Lin Z, Ko YH, Goldberg AF, Flomenberg N, Wang C, Pavlides S, Pestell RG, Howell A, Sotgia F, Lisanti MP. Understanding the metabolic basis of drug resistance: therapeutic induction of the Warburg effect kills cancer cells. Cell Cycle. 2011; 10:2521-28.

12. Györffy B, Lanczky A, Eklund AC, Denkert C, Budczies J, Li Q, Szallasi Z. An online survival analysis tool to rapidly assess the effect of 22,277 genes on breast cancer prognosis using microarray data of 1,809 patients. Breast Cancer Res Treat. 2010; 123:725-31.

13. Fiorillo M, Sotgia F, Sisci D, Cappello AR, Lisanti MP. Mitochondrial "power" drives tamoxifen resistance: NQO1 and GCLC are new therapeutic targets in breast cancer. Oncotarget. 2017; 8:20309-27. https://doi.org/10.18632/ oncotarget. 15852 .

14. Lamb R, Harrison H, Hulit J, Smith DL, Lisanti MP, Sotgia F. Mitochondria as new therapeutic targets for eradicating cancer stem cells: quantitative proteomics and functional validation via MCT1/2 inhibition. Oncotarget. 2014; 5:11029-37. https://doi.org/10.18632/oncotarget.2789.

15. Lamb R, Ozsvari B, Lisanti CL, Tanowitz HB, Howell A, Martinez-Outschoorn UE, Sotgia F, Lisanti MP. Antibiotics that target mitochondria effectively eradicate cancer stem cells, across multiple tumor types: treating cancer like an infectious disease. Oncotarget. 2015; 6:4569-84. https://doi. org/10.18632/oncotarget.3174.

16. Farnie G, Sotgia F, Lisanti MP. High mitochondrial mass 
identifies a sub-population of stem-like cancer cells that are chemo-resistant. Oncotarget. 2015; 6:30472-86. https://doi. org/10.18632/oncotarget.5401.

17. Fiorillo M, Lamb R, Tanowitz HB, Mutti L, KrsticDemonacos M, Cappello AR, Martinez-Outschoorn UE, Sotgia F, Lisanti MP. Repurposing atovaquone: targeting mitochondrial complex III and OXPHOS to eradicate cancer stem cells. Oncotarget. 2016; 7:34084-99. https:// doi.org/10.18632/oncotarget.9122.

18. De Luca A, Fiorillo M, Peiris-Pagès M, Ozsvari B, Smith DL, Sanchez-Alvarez R, Martinez-Outschoorn UE, Cappello AR, Pezzi V, Lisanti MP, Sotgia F. Mitochondrial biogenesis is required for the anchorage-independent survival and propagation of stem-like cancer cells. Oncotarget. 2015; 6:14777-95. https://doi.org/10.18632/ oncotarget.4401.

19. Fiorillo M, Lamb R, Tanowitz HB, Cappello AR, MartinezOutschoorn UE, Sotgia F, Lisanti MP. Bedaquiline, an FDA-approved antibiotic, inhibits mitochondrial function and potently blocks the proliferative expansion of stem-like cancer cells (CSCs). Aging (Albany NY). 2016; 8:1593607. https://doi.org/10.18632/aging. 100983.

20. Martinez-Outschoorn UE, Peiris-Pagés M, Pestell RG, Sotgia F, Lisanti MP. Cancer metabolism: a therapeutic perspective. Nat Rev Clin Oncol. 2017; 14:11-31.
21. Lamb R, Fiorillo M, Chadwick A, Ozsvari B, Reeves KJ, Smith DL, Clarke RB, Howell SJ, Cappello AR, MartinezOutschoorn UE, Peiris-Pagès M, Sotgia F, Lisanti MP. Doxycycline down-regulates DNA-PK and radiosensitizes tumor initiating cells: implications for more effective radiation therapy. Oncotarget. 2015; 6:14005-25. https:// doi.org/10.18632/oncotarget.4159.

22. Bonuccelli G, Peiris-Pages M, Ozsvari B, MartinezOutschoorn UE, Sotgia F, Lisanti MP. Targeting cancer stem cell propagation with palbociclib, a CDK4/6 inhibitor: telomerase drives tumor cell heterogeneity. Oncotarget. 2017; 8:9868-84. https://doi.org/10.18632/ oncotarget.14196.

23. Peiris-Pagès $M$, Martinez-Outschoorn UE, Pestell RG, Sotgia F, Lisanti MP. Cancer stem cell metabolism. Breast Cancer Res. 2016; 18:55.

24. Bonuccelli G, De Francesco EM, de Boer R, Tanowitz HB, Lisanti MP. NADH autofluorescence, a new metabolic biomarker for cancer stem cells: identification of Vitamin $\mathrm{C}$ and CAPE as natural products targeting "stemness". Oncotarget. 2017; 8:20667-78. https://doi.org/10.18632/ oncotarget. 15400 . 